\title{
Systematic study of hydrogenation in a diamond amplifier
}

\author{
Erdong Wang, ${ }^{1}$ Ilan Ben-Zvi, ${ }^{2,3}$ Xiangyun Chang, ${ }^{2}$ Qiong Wu, ${ }^{2}$ Triveni Rao, ${ }^{4}$ John Smedley, ${ }^{4}$ \\ Jorg Kewisch, ${ }^{2}$ and Tianmu Xin ${ }^{3}$ \\ ${ }^{1}$ State Key Laboratory of Nuclear Physics and Technology, Institute of Heavy Ion Physics, School of Physics, Peking University, \\ Beijing 100081, China \\ ${ }^{2}$ Collider Accelerator Department, Brookhaven National Laboratory, Upton, New York 11973, USA \\ ${ }^{3}$ Physics and Astronomy Department, Stony Brook University, Stony Brook, New York 11974, USA \\ ${ }^{4}$ Instrumentation Division, Brookhaven National Laboratory, Upton, New York 11973, USA
}

(Received 11 February 2011; published 21 June 2011)

\begin{abstract}
We recently developed a reliable hydrogenation procedure for the diamond amplifier that assures the generation of a high-current, high-brightness beam. In this paper, we compare room-temperature hydrogenation with that at high temperatures. We identified the factors leading to the decay of quantum efficiency. The optimum temperature for heat treatment ranged from $400-450^{\circ} \mathrm{C}$; its superiority was proven in the gain test. Hydrogenated diamond amplifiers exposed to $\mathrm{N}_{2}$ and air exhibited a good emission after being heated to $350^{\circ} \mathrm{C}$; the highest gain we registered in emission scanning was 178 . Our systematic study of hydrogenation resulted in the reproducible fabrication of diamond amplifiers.
\end{abstract}

DOI: 10.1103/PhysRevSTAB.14.061302

PACS numbers: 29.25.Bx

\section{INTRODUCTION}

Assuring a high average-current, high-brightness, and low-emittance electron beam is a key technology for modern accelerator-based science, such as energy-recovery linac light sources [1], and electron cooling of hadron accelerators [2]. The high average currents generation from traditional photocathodes directly requires high quantum efficiency $(\mathrm{QE})$ cathodes and very powerful lasers. The diamond-amplified photocathode has demonstrated excellent performance for an electron source that promises to support a high average current, low emittance, and highly stable electron beam with a long lifetime [3]. The diamond, functioning as a secondary emitter, amplifies the primary current of a few keV energy that comes from a laser-drive photocathode and a DC structure. The surface of one side of the diamond is coated with a metal, like Pt, while its other side is hydrogenated to attain a negative electron-affinity surface [4]. Primary electrons penetrate the diamond through the metal coating, and excite electron-hole pairs, the number of which typically is about 2 orders of magnitude more than the number of primary electrons, depending on their energy. Secondary electrons drift across the diamond under an electric field provided by the gun's electrical field that penetrates the diamond. The holes drift back to the metal side and are absorbed by the ground as the secondary electrons reach the hydrogenated surface and exit into the vacuum through the diamond's negative electron-affinity (NEA) surface.

Published by the American Physical Society under the terms of the Creative Commons Attribution 3.0 License. Further distribution of this work must maintain attribution to the author(s) and the published article's title, journal citation, and DOI.
In this article, we describe our optimization of the hydrogenation process for the diamond amplifier that resulted in a stable emission gain of 140 , more than the maximum gain of 40 achieved in our previous emission experiment [5]. These characteristics of our diamond amplifiers are reproducible. We demonstrate the robustness of the diamond's NEA surface.

\section{HYDROGENATION EXPERIMENTS}

We carried out the hydrogenation experiments in a bakable UHV chamber evacuated to $1.7 \times 10^{-9} \mathrm{hPa}$ by a turbo pump and an ion pump. The chamber was equipped with a residual gas analyzer and a hydrogen cracker. The diamond, biased to $-50 \mathrm{~V}$, was placed on a button heater to heat it to $800^{\circ} \mathrm{C}$. A thermocouple, in thermal contact with the sample's base, monitored the temperature. The diamond was illuminated directly with a deuterium lamp that has a continuous emission spectrum between 190 to $300 \mathrm{~nm}$. The strongest emission is at $230 \mathrm{~nm}$ wavelength with a power of $2.46 \mu \mathrm{W}$. The lamp was coupled to a UV monochromator, enabling us to acquire the spectral dependence of photoemission in the same spectral range. The diameter of the UV light's spot was $3 \mathrm{~mm}$, covering the entire hydrogenated surface of the diamond. To fabricate a diamond amplifier, we Pt coated one side of high purity $4 \times 4 \mathrm{~mm}^{2}, \quad 300 \mu \mathrm{m}$-thick single-crystal diamond samples, grown by chemical deposition; the other side was hydrogenated. The steps in fabricating diamond amplifiers were as follows: (i) The diamond sample was cleaned ultrasonically in acetone and then in $100 \%$ alcohol for 15 minutes. (ii) $35 \mathrm{~nm} \mathrm{Pt}$ was sputtered onto one side of the diamond wafer. (iii) The diamond was heated to $800^{\circ} \mathrm{C}$ to clean its surface. (iv) A UV ray was shone on the prospective hydrogenated surface, and the photocurrent 
was measured. (v) The sample was exposed to a flow of hydrogen atoms generated by a commercial hydrogen cracker. (vi) After the photocurrent reached its peak, the source of hydrogen atoms was turned off. (vii) Fabrication of the diamond amplifier was complete after its temperature had dropped to room temperature.

One important parameter of the hydrogenated diamond is its photoemission QE that is an indicator of the negative affinity of its surface.

We compared four diamonds hydrogenated at room temperature with four others treated at high temperatures. For the latter, after temperature of the diamond reached $800^{\circ} \mathrm{C}$, the heater was turned off; hydrogenation was started, and continued as the sample's temperature decreased gradually to $320^{\circ} \mathrm{C}$. For room-temperature hydrogenation, the sample was allowed to cool down to $23^{\circ} \mathrm{C}$ before starting hydrogenation. For both, we set the power of the hydrogen cracker at $50 \mathrm{~W}$, and the hydrogen partial pressure was $1.3 \times 10^{-6} \mathrm{hPa}$. The electron yield was monitored while the $220 \mathrm{~nm}, 2.25 \mu \mathrm{W}$ UV ray was shone on the diamond.

Figure 1 shows a typical curve for photocurrent yield from the hydrogenated surface of sample treated at $800^{\circ} \mathrm{C}$ (dark curve) and at $23^{\circ} \mathrm{C}$ (gray curve).

As Fig. 1 shows, the photocurrent took 30 minutes to reach a peak when the diamond was hydrogenated at high temperature; in contrast, during hydrogenation at $23^{\circ} \mathrm{C}$, the photocurrent peaked in 10 minutes. The hydrogenation process started immediately. The speed of hydrogen deposition differed at these two different temperatures. At high temperatures, hydrogen attaches to and detaches from the carbon atoms. Hence, it takes longer to reach optimum coverage than when the process is carried out at room temperature at which the detachment of hydrogen is insignificant. Further hydrogenation does not increase the coverage. However, it exposes the sample to contaminants released from the cracker that may cause impingement on the diamond's NEA surface causing the photocurrent to decay. This reduction was unrecoverable by subsequent

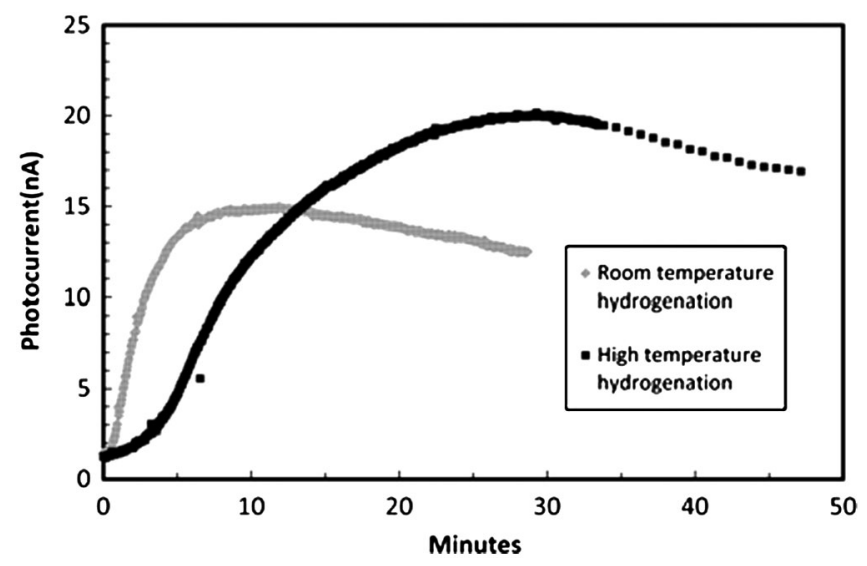

FIG. 1. The trend in the photocurrent during the hydrogenation process. The dark curve represents the trend during high-temperature hydrogenation, and the gray curve is that at room temperature.

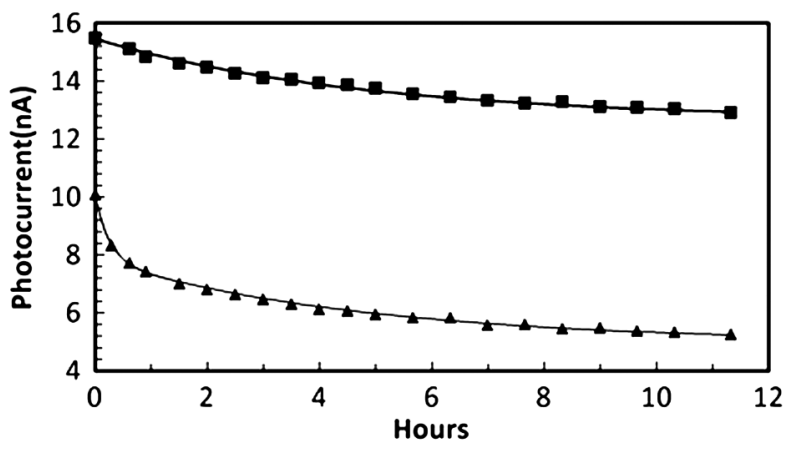

FIG. 2. The stabilization of the photocurrent of hydrogenated diamonds in 11 hours. The solid square is the photocurrent of the high-temperature-treated diamond; the solid triangle is the photocurrent of hydrogenation decay at room temperature. The thin black curves are the best fit functions.

rebaking or rehydrogenation. Figure 2 shows the QE decays of the high-temperature and room-temperature hydrogenation process. At the end of hydrogenation (after the cracker was turned off and the hydrogen pumped from the system), the change in QE over time was measured with $220 \mathrm{~nm}$ beam. In 11 hours, the QE of the diamond processed at high temperature dropped $13 \%$, while that of the diamond treated at room temperature declined 50\%; thus, the NEA surface produced via high-temperature hydrogenation is more stable than that created at room temperature. The high-temperature hydrogenation decay curve is best fit to the function $I(t)=12.7[\mathrm{nA}]+2.8[\mathrm{nA}] \exp (-t / 4.8[\mathrm{~h}])$, while the best fit for the decay after room-temperature hydrogenation is a twin decay function, $I(t)=5.0[\mathrm{nA}]+2.9[\mathrm{nA}] \times$ $\exp (-t / 4.8[\mathrm{~h}])+2.2[\mathrm{nA}] \exp (-t / 0.3[\mathrm{~h}])$. Therefore, the decay curve of the latter has two components, one with a decay time of 0.3 hours, and a slow component where the decay time ( $\sim 4.8$ hours) is common to both processes curves. Such loss of QE can be recovered by baking the sample. Thus, after the decay of the QE in 11 hours, we baked the diamonds at $400^{\circ} \mathrm{C}$ for 30 minutes. There was almost full recovery (99\%) of the QE of the diamond that underwent high-temperature hydrogenation; the decay of the photocurrent under this condition is due to contaminants, such as water absorbed on the hydrogenated surface that are desorbed to the surface during baking [6]. However, the QE of the diamond hydrogenated at the room temperature exhibited only $65 \%$ recovery after baking, implying that baking can correct the slow decay, but not that lost during the fast decay.

Several factors might cause the QE decay of the hydrogenated diamond including interactions between the UV beam, residual gas, and the diamond surface, or the contamination of the diamond's surface by residual gas. Surfaces contaminated by the back bombardment of the ions generated by the UV light decompose the residual gas, as similarly, does the back bombardment of ions generated by photoemission electron ionization. Identifying the causes of photocurrent decay undoubtedly would improve 


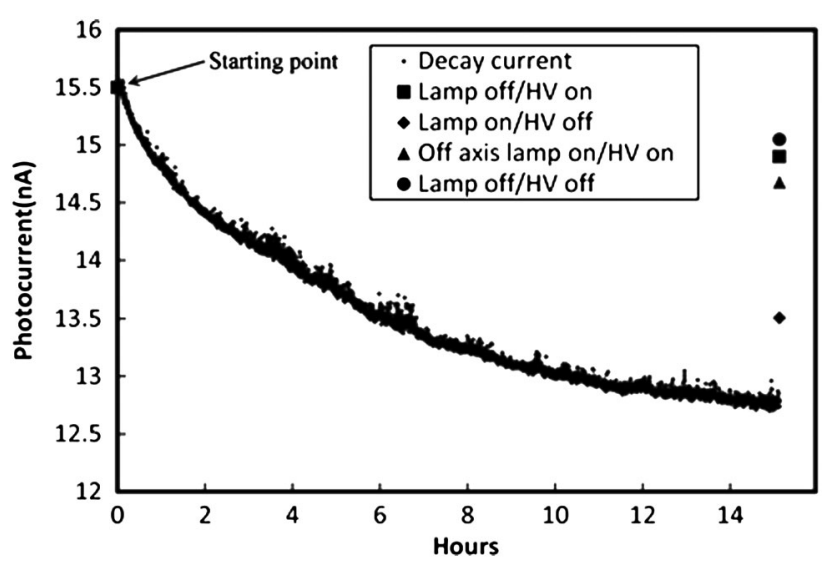

FIG. 3. The stabilization of the photocurrent measured in the diamond sample under different conditions.

the hydrogenation process. The power of the UV light was $2.25 \mu \mathrm{W}$ at $220 \mathrm{~nm}$, and the diamond was biased to $-50 \mathrm{~V}$. In this experiment, the base pressure of the high vacuum chamber was $2 \times 10^{-9} \mathrm{hPa}$. Figure 3 shows the photocurrent decay under different conditions. During every cycle of the measurement, we first heated the diamond sample to $800^{\circ} \mathrm{C}$ for 30 minutes to obtain a bare surface before following the high-temperature hydrogenation procedure. The decay in photoemission was measured under various conditions. The total decline of $\mathrm{QE}$ in 15 hours is $18 \%$. We assumed, for simplicity, that in the following the processes were additive. Then, we summed the effects and derived values for them individually to explain which contributed to causing a decay of $13.8 \%$.

(a) When the UV light and the bias voltage were off, the residual gas contaminated the hydrogenation surface. Over 15 hours, the photocurrent dropped by $1.9 \%$.

(b) When the UV light was on and there was no bias voltage, the residual gas and the UV light together affected the hydrogenated surface. In 15 hours, the photocurrent fell by $11.7 \%$. After subtracting the decay due to residual gas described in (a) above, the photocurrent decrease due to the UV light was $9.8 \%$.

(c) When the UV light was off and the bias voltage was on, the positive ions in the chamber were back bombarded and impinged on this surface, and the residual gas contaminated it simultaneously so that in 15 hours, the QE dropped by $2.6 \%$. After removing the decay due to residual gas as in (a) above, the photocurrent decayed by $0.7 \%$ due to the bias voltage.

(d) To study the effect of the UV light on the residual gas and its impact on the QE, the UV light was shifted to irradiate the holder and not the diamond surface, keeping the bias voltage on. Then, the residual gas still would be ionized by UV and the ions would bombard the diamond's surface. This process lowered the QE by $4 \%$. After subtracting the decays described in (a) and (c) above, we conclude that the photocurrent fell by $1.4 \%$ due to the ions generated by the UV light, so leading to back bombardment.
TABLE I. Different causes of photocurrent decay.

\begin{tabular}{ll}
\hline \hline Decay factor & $\begin{array}{l}\text { Decay rate of } \\
\text { photocurrent }(\%)\end{array}$ \\
\hline Residual gas & 1.9 \\
UV light, no bias & 9.8 \\
UV light ionized gas back bombardment & 1.4 \\
Bias voltage & 0.7 \\
Probable emission electron ionization & 4.2 \\
residual gas ion back bombardment & \\
All & 18 \\
\hline \hline
\end{tabular}

Assuming that these reductions are additive, and taking into account the overlapping processes, and then the three processes detailed above explain a decay of $13.8 \%$. The reason of the additional $4.2 \%$ decline in 15 hours was unknown.

To identify this yet unexplained reduction, we changed the background vacuum to $3.8 \times 10^{-9} \mathrm{hPa}$; then, this unidentified part of the decay increased to $7.2 \%$, and depends on the background vacuum. Therefore, this part of the decay in the photocurrent decay may reflect the back bombardment of ions generated by the ionization of photoemitted electrons. If these different factors independently cause the decay of the diamond's QE, then we can identify the factors affecting its photocurrent decay (Table I).

The diamond amplifier is extremely robust and is stable during exposure to air; the water vapor in the air inhibits electron emission from it [6]. Heating diamonds exposed to the atmosphere removes water molecules from their surfaces. We explored the optimal temperature for such evaporation; the photocurrent of the diamond amplifier with a new hydrogenation surface is $17 \mathrm{nA}$. After exposure to air for $1 \mathrm{~h}$, the emission current falls to $2 \mathrm{nA}$. We then heated the diamond to $200^{\circ} \mathrm{C}$ for 30 minutes and left it cool. Our measurements of the photocurrent show the

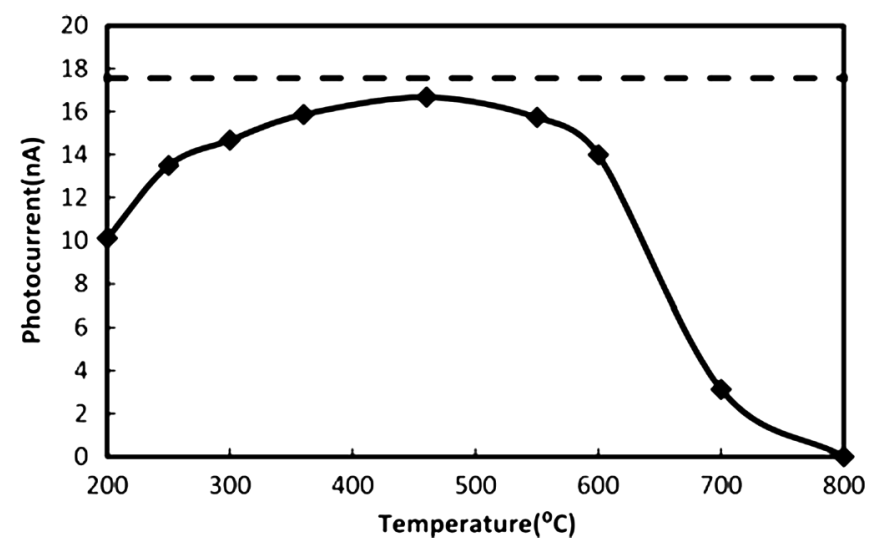

FIG. 4. Temperature scanning for optimizing heat treatment of the diamond. The dashed line is the photocurrent of a freshly hydrogenated diamond. The solid curve is the photocurrent after heating the sample to the temperature indicated and allowing it to cool down. 
diamond's photocurrent rebounded to $10 \mathrm{nA}$. We scanned the $\mathrm{QE}$ as a function of the temperature of the heat treatment: Figure 4 shows that the optimized temperature for heat treatment is $450^{\circ} \mathrm{C}$ after which the photocurrent recovered to $96 \%$ of that of an amplifier unexposed to the atmosphere. The findings prove that the quality of hydrogenation is recovered by baking. Temperatures higher than $450^{\circ} \mathrm{C}$ break the hydrogen and carbon bonds. At $800^{\circ} \mathrm{C}$, hydrogen atoms are removed from the diamond surfaces, leaving it bare.

\section{GAIN MEASUREMENT}

Our setup for measuring the emission-mode gain consists of a thermionic gun, a high-voltage pulsed-power supply, an anode, and a phosphor screen; details are published elsewhere [7]. The thermionic gun supplies the DC primary electrons that penetrate the metal layer. There is a gap of 200 to $500 \mu \mathrm{m}$ between the anode and the diamond's hydrogenation surface. The applied high voltage between the anode and the metalized surface of the diamond generates a field in the diamond and across the gap, towards which electrons emitted from the diamond are accelerated. The average value of the emission current is measured by the integrated anode current. The electrometer grounds the anode. The emission gain is defined as the ratio of the collected current on the anode to the primary electron current.

The hydrogenation chamber and emission-mode gain test chamber are separate. During transfer from the hydrogenation chamber to the test chamber, the freshly hydrogenated sample is exposed to ambient air for 30 minutes, during which the surface absorbs water molecules. We measured the gain at the center of the diamond amplifier after a $350^{\circ} \mathrm{C}$ heating/cooling cycle. We then exposed the amplifier to dry $\mathrm{N}_{2}$ and to air at $1.01 \times 10^{4} \mathrm{hPa}$ for 30 minutes. Following another $350^{\circ} \mathrm{C}$ heating and cooling cycle, we noted that the gain dropped by $3 \%$ after $\mathrm{N}_{2}$ exposure, and by $7 \%$ after exposure to atmospheric air. We transferred the diamond from the hydrogenation chamber to the gain test chamber in a nitrogen environment instead of exposing it to air. This did not change the reduction in gain before heating.

Next, a freshly hydrogenated diamond amplifier, only briefly exposed to the air, was installed in the test chamber. It was heated to $200^{\circ} \mathrm{C}$ under an ultrahigh vacuum $\left(\sim 10^{-10} \mathrm{hPa}\right)$, held there for 40 minutes, and then allowed to cool to room temperature. The gain at the center of the amplifier was 95 . Heating it there for 40 minutes at $350^{\circ} \mathrm{C}$ (the limit in our test chamber) gave a gain of 135 at the same location upon cooling. This result shows that the optimum temperature for heat treatment is much higher than $200^{\circ} \mathrm{C}$.

Our studies resulted in optimized parameters for hydrogenation and baking, giving us diamond samples with reproducible NEA surfaces, high $\mathrm{QE}$, and high gain. We

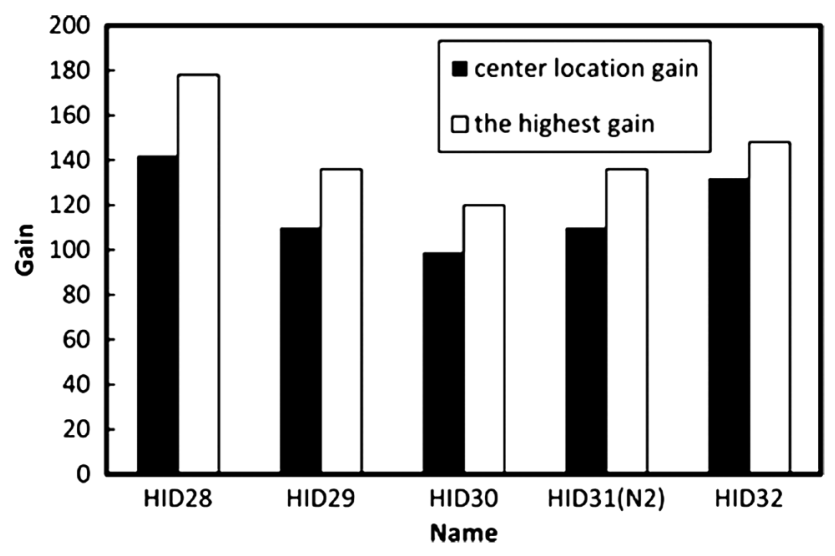

FIG. 5. The gain in five different diamond amplifiers. HID 31 was transferred in dry $\mathrm{N}_{2}$ protection from the hydrogenation system to the gain test system. The black columns show the gain of the centers of the diamond amplifiers, and the white columns show the maximum gain on the samples.

fabricated five more samples, and the gain in all of them reached more than 100. Diamond amplifiers are driftdominated cathodes, so the diameter of secondary electron beam reaching the emission surface is the same as that of the primary bunch. The primary electron beam's size was minimized to $0.5 \mathrm{~mm}^{2}$; we used it to scan the diamond amplifier. The highest emission gain we measured was 178 . Figure 5 shows the gain in each of the five amplifiers we fabricated. The different gain at different locations within the whole piece could be due to the nonuniformities in the metal coating. The primary electrons will deposit part of their energy in the metal coating before penetrating the diamond. The lost energy varies with the thickness of the metal coating. The residual energy of primary electron affects the gain of the amplifier.

\section{SUMMARY}

We studied the effect of hydrogenation on the NEA surface of diamond amplifiers, finding that hightemperature hydrogenation yields a higher quality NEA surface compared to the hydrogenation at room temperature. Hydrogenated diamond amplifiers are little affected by exposure to the atmosphere; any loss in electron yield can be recovered by subsequent heat treatment. We optimized the bake temperature to recover the maximum electron yield in both the hydrogenation chamber and in the test chamber. Our treatments result in a reproducibly better performance of diamond amplifiers.

\section{ACKNOWLEDGMENTS}

This work was carried out at Brookhaven Science associates, LLC under Contracts No. DE-AC02-98CH10886 and at Stony Brook University under Grant No. DESC0005713 with the U.S. DOE. 
[1] S. M. Gruner et al., Rev. Sci. Instrum. 73, 1402 (2002).

[2] Ya. S. Derbenev, Nucl. Instrum. Methods Phys. Res., Sect. A 441, 223 (2000).

[3] I. Ben-Zvi et al., Secondary Emission Enhanced Photoinjector (Brookhaven National Laboratory, Upton, 2004), Vol. C-A/AP/149 [http://www.agsrhichome.bnl .gov/AP/ap_notes/ap_note_149.pdf].
[4] J. van der Weide et al., Phys. Rev. B 50, 5803 (1994).

[5] X. Chang et al., Phys. Rev. Lett. 105, 164801 (2010).

[6] G. Piantanida et al., J. Appl. Phys. 89, 8259 (2001).

[7] X. Chang et al., in Proceedings of the 23rd Particle Accelerator Conference, Vancouver, Canada, 2009 (IEEE, Piscataway, NJ, 2009), p. 691. 